\title{
Orthobunyaviruses: recent genetic and structural insights
}

\section{Richard M. Elliott}

Abstract | Orthobunyaviruses, which have small, tripartite, negative-sense RNA genomes and structurally simple virions composed of just four proteins, can have devastating effects on human health and well-being, either by causing disease in humans or by causing disease in livestock and crops. In this Review, I describe the recent genetic and structural advances that have revealed important insights into the composition of orthobunyavirus virions, viral transcription and replication and viral interactions with the host innate immune response. Lastly, I highlight outstanding questions and areas of future research.

Orthobunyaviruses belong to the Bunyaviridae family, which is the largest and most diverse family of RNA viruses, comprising more than 350 named isolates. These negative-sense RNA viruses take their name from Bunyamwera virus (BUNV), which was originally isolated in 1943 from Aedes spp. mosquitoes during an investigation of yellow fever in the Semliki Forest, Uganda. During the following 25 years, several antigenically related viruses were identified in laboratories in South America, Africa and India, mostly owing to work that was funded by The Rockefeller Foundation ${ }^{1}$. The serological relationships between these isolates initially led to the Bunyamwera supergroup of viruses, until further biochemical and structural information resulted in the formal establishment of the family Bunyaviridae in 1975. The family currently contains five genera: Orthobunyavirus, Hantavirus, Nairovirus, Phlebovirus and Tospovirus. Orthobunyaviruses, nairoviruses and phleboviruses are all arboviruses, which means that they replicate in, and are transmitted by, blood-feeding arthropods (such as mosquitoes or ticks), but they can also infect vertebrates. Tospoviruses are also transmitted by arthropods (in this case, thrips), but they infect plants, such as tomato and watermelon, rather than vertebrates. Hantaviruses are mainly carried by rodents, but humans can be infected via aerosolized rodent excreta $^{2}$.

At least 30 orthobunyaviruses are responsible MRC-University of Glasgow Centre for Virus Research, 464 Bearsden Road, Glasgow G61 1OH, UK e-mail: richard.elliott@glasgow.ac.uk doi: 10.1038/nrmicro3332 Published online

8 September 2014 an increasing awareness of the potential detrimental effects of orthobunyaviruses on human and animal health, we know little about the burden of orthobunyavirus disease; for example, it is estimated that there are several hundred thousand asymptomatic LACV infections annually ${ }^{4}$ in the United States, but the true number of infections is unknown.

In 2011, a novel orthobunyavirus, Schmallenberg virus (SBV), emerged in Europe (BOX 1), which emphasizes the potential of these viruses to emerge in new localities and cause serious disease. In addition, the structures of orthobunyavirus nucleocapsid proteins and virus particles have recently been determined, which has generated renewed interest in these viruses. In this Review, I discuss these structural advances, which, together with exploitation of reverse genetics to probe orthobunyavirus replication and interactions with host innate immune responses, are providing new insights into orthobunyavirus biology.

\section{Arthropod transmission}

Most orthobunyaviruses are transmitted by mosquitoes or culicoid flies (that is, midges), although a few are vectored by ticks and the Kaeng Khoi virus is transmitted by bed bugs ${ }^{3}$. In general, only one or a very few arthropod species are competent to transmit a particular orthobunyavirus, and strict vector preference is observed even in regions where different viruses and vectors coexist $^{5}$. Female mosquitoes acquire the virus when feeding on an infected host, and infected mosquitoes show few adverse signs of infection ${ }^{6}$, although changes in the feeding behaviour of LACV-infected Aedes spp. mosquitoes have been detected ${ }^{7}$ and LACV-infected female mosquitoes have been reported to mate more efficiently than their uninfected counterparts ${ }^{8}$. Mosquitoes become 


\section{Box 1 An emerging orthobunyavirus in Europe: Schmallenberg virus}

In the summer of 2011, a relatively mild disease in cattle was reported in farms in Germany and the Netherlands, which had symptoms of transient fever, diarrhoea and reduced milk production. Using metagenomics, researchers identified a novel orthobunyavirus as the cause, which was named Schmallenberg virus (SBV) after the town in which it was first isolated ${ }^{115}$. Later that year, reports of abortions, stillbirths and offspring with congenital abnormalities - such as arthrogryposis and hydranencephaly - were recorded in cattle, sheep and goat herds in western Europe ${ }^{116}$, and SBV RNA was detected in the brains of affected foetuses. During 2012 and 2013, SBV spread rapidly throughout Europe; the disease was recorded in 27 European countries and involved $>8,000 \mathrm{farms}^{111,117}$. The overall economic impact of the SBV epidemic is considered to be low by the European Union ${ }^{118}$, but the effects on individual farms have been devastating, and some farmers reported more than $50 \%$ losses of lambs ${ }^{119}$.

$\mathrm{SBV}$ is related to orthobunyaviruses of the Simbu serogroup, which includes Aino virus, Akabane virus, Sathuperi virus and Shamonoda virus ${ }^{120}$, which cause a similar disease in ruminant offspring in Africa, Asia and Australia. Similarly to these viruses, SBV was shown to be transmitted by Culicoides spp. midges ${ }^{121}$, and the wind-borne dispersal of infected midges (including across the English Channel) had a major role in the spread of the virus ${ }^{122}$.

Research on SBV has progressed at a rapid pace. A PCR test for rapid diagnosis was quickly developed ${ }^{123}$ and reverse genetic systems were established ${ }^{13,63}$ (BOX 2), which will facilitate studies on SBV molecular biology and pathogenesis. The crystal structure of the SBV N (nucleocapsid) protein was reported in 2013, which was the first orthobunyavirus $\mathrm{N}$ protein structure to be determined $\mathrm{d}^{46,47}$. In addition, two inactivated virus vaccines have been developed and licensed for use in Europe ${ }^{113}$. However, many questions remain unanswered. First, it is unclear where the virus originated, but it has been proposed that it could have reached Europe via the flower trade from East Africa to the Netherlands ${ }^{124}$. It is also unclear how SBV survives over the winter when vector activity is minimal, although vertical transmission in midges has been suggested ${ }^{125}$. Immunity in cattle lasts at least 1 year ${ }^{126}$, but this has not yet been determined for sheep; such information is required to predict the future impact of SBV and to advise on vaccination strategies. Last, how will SBV evolve? Variants of SBV have been reported ${ }^{127,128}$, and a hot spot for mutation has been detected in the Gc protein ${ }^{129,130}$, so it will be interesting to determine whether these mutations affect pathogenicity and the ability to escape pre-existing immunity.

Arthrogryposis

A congenital abnormality in which the limb joints are deformed and inflexible.

\section{Hydranencephaly}

A condition that arises during

foetal development, in which the central cavity of the brain is missing and is replaced with cerebrospinal fluid.

\section{Complement fixation} An immunological test to measure whether antibodyantigen complexes can deplete a known concentration of standard complement proteins and protect added red blood cells from lysis. For orthobunyaviruses, complement-fixing antibodies are elicited by the nucleocapsid protein and are used to define serogroups. persistently and systemically infected, and in some cases, vertical (that is, transovarial) transmission to offspring has been reported ${ }^{5}$. Transovarial transmission is an important mechanism for the maintenance of some viruses (such as LACV) during the winter months and can result in infected male mosquitoes that transfer the virus to females during mating ${ }^{9}$. The mild nature of the infection is clearly advantageous for mosquito transmission, and both horizontal and vertical transmission between mosquitoes obviates the need for a vertebrate host, which suggests that orthobunyaviruses might have initially evolved as insect-only viruses. Indeed, the recent isolation of novel viruses from different mosquito species that form a phylogenetic sister clade to orthobunyaviruses (such as Herbert virus and related viruses) and that seem to be unable to replicate in vertebrate cells, provides support for this hypothesis ${ }^{10}$.

\section{Pathogenesis in the vertebrate host}

Infection is initiated by the bite of an infected arthropod and the virus spreads to striated muscle, where extensive replication occurs, which results in high viraemia. The virus then spreads to most organs of the body and can cross the blood-brain barrier, where it targets neurons ${ }^{11}$; infection of the central nervous system (CNS) is agerelated and juvenile animals seem to be more susceptible than adults. However, studies of LACV-infected mice have shown that the virus remains neurovirulent (that is, it causes a lethal infection following direct intracranial inoculation), even when neuroinvasiveness (that is, spread to the CNS following peripheral inoculation) in older animals is lost ${ }^{12}$. Transfer to the CNS might also be mediated by olfactory neurons, which was demonstrated in weanling mice that were infected with $\mathrm{LACV}^{12}$. Examination of malformed lambs and calves that were born from SBV-infected animals has also shown that viral antigens reach high levels in brain tissue ${ }^{13}$, and similar findings have been reported for other orthobunyaviruses that cause teratogenic effects. In sheep that are experimentally infected with SBV, the period of viraemia is short (3-5 days), but viral RNA is detected in the spleen and lymph nodes for up to 44 days $^{14}$; the importance of persistence in the lymphoreticular system requires further investigation.

The incubation period of orthobunyaviruses in humans is typically 3-8 days, followed by the sudden onset of fever, headache, joint and muscle aches, which are occasionally accompanied by nausea and rash. Symptoms usually last for around 1 week, and a second bout of fever may occur 1-2 weeks later (particularly with OROV infection). Although most patients recover without long-lasting sequelae, severe LACV infections can result in seizures and convulsions that can last for up to 1 year. In addition, a few fatalities owing to LACV infection have been reported ${ }^{15}$.

\section{Molecular biology of orthobunyaviruses}

There are more than 170 named viruses in the Orthobunyavirus genus, which are grouped into 18 serogroups on the basis of serological relatedness, as determined by complement fixation, neutralization and haemagglutination inhibition assays ${ }^{3}$. Few of these viruses have been studied at the molecular level, and much of what is known about orthobunyavirus molecular biology is based on the study of only a few viruses, predominately BUNV (which is the prototype of both the genus and family) and LACV. However, as more information becomes available for different viruses, it is clear that there is great diversity among orthobunyaviruses, and it is highly likely that there will be exceptions to the generalizations that are outlined below.

Virus particle. Orthobunyavirus virions are relatively simple in their overall composition and comprise just four structural proteins: two surface glycoproteins, termed Gn and Gc, and two internal proteins, the $\mathrm{N}$ (nucleocapsid) protein and the L protein (the viral RNA-dependent RNA polymerase (RdRp)). The three genomic RNA segments are encapsidated by the N protein to form ribonucleoprotein (RNP) complexes that associate with the RdRp and are contained within the lipid envelope of the particle (FIG. 1a). Early electron microscopy analyses of purified virions showed spherical to pleomorphic particles that were $\sim 90 \mathrm{~nm}$ in diameter, with a double membrane envelope and a fringe of spikes that were predicted to be the glycoproteins ${ }^{16}$. Recent cryoelectron microscopy studies and 


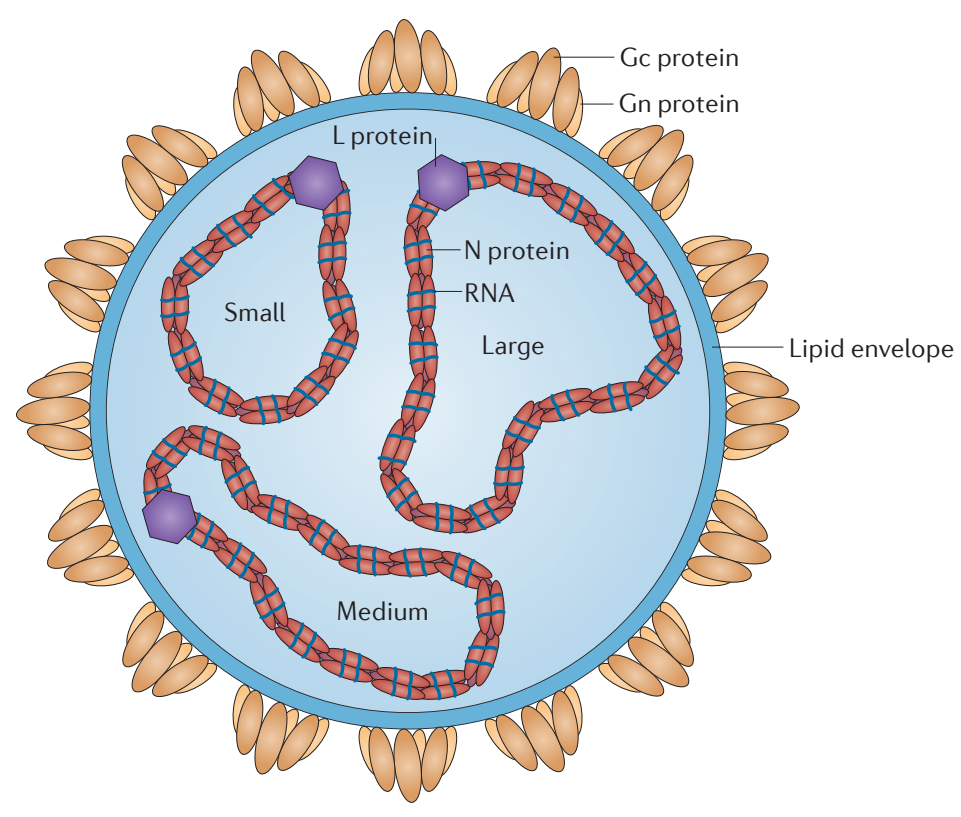

b

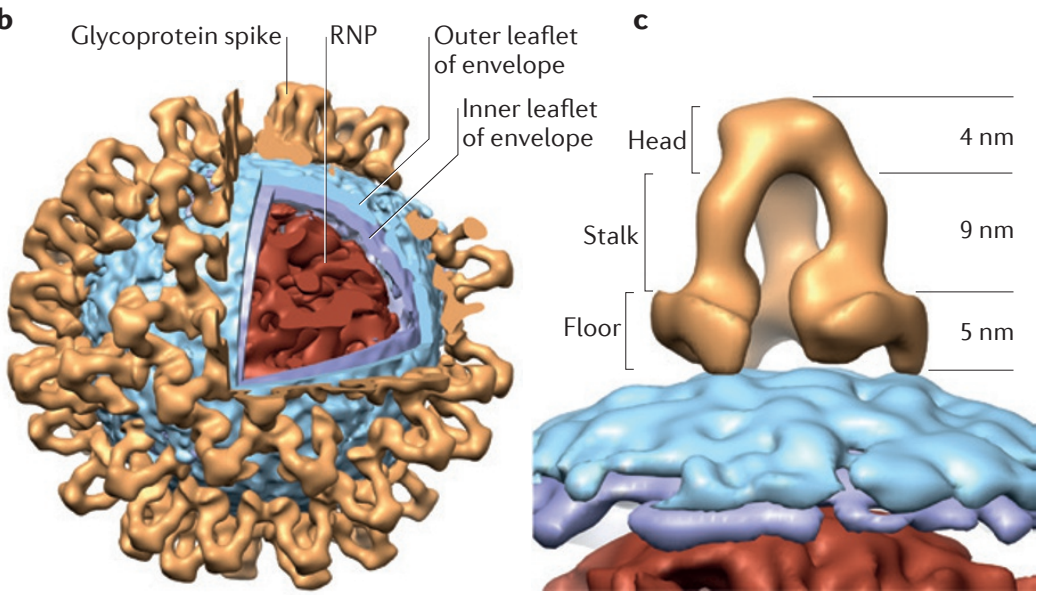

Figure 1 | The orthobunayvirus particle. a|Schematic representation of the orthobunyavirus virion. The three genome segments (small, medium and large) are encapsidated by the $\mathrm{N}$ (nucleocapsid) protein to form ribonucleoprotein (RNP) complexes, which associate with the viral RNA-dependent RNA polymerase (RdRp). The RNPs are packaged within a lipid envelope that is derived from the host cell Golgi complex, which is modified by insertion of the viral glycoproteins $\mathrm{Gn}$ and Gc. The Gn and Gc proteins form a trimer of heterodimers that create the spikes on the virus particle. b |A cross-section model of a Bunyamwera virus virion based on a tomographic reconstruction. c| The tripodal architecture of the glycoprotein spike can be divided into two major protein contacts: the floor region, which is adjacent to the membrane; and the membrane-distal head region. These two protein contacts are separated from each other by a stalk region. Structural regions and their corresponding dimensions are indicated. Parts $\mathbf{b}$ and $\mathbf{c}$ of the figure are reproduced from REF. 17.

\section{Neutralization}

An immunological test to detect antibodies that bind to

a virus and reduce its titre in an infectivity assay. For

orthobunyaviruses, neutralizing antibodies are elicited by the viral glycoproteins and are used for the identification of specific viruses. subtomographic averaging have revealed much greater detail of the structure of BUNV ${ }^{17}$. Virions average $108 \pm 8 \mathrm{~nm}$ in diameter and, although they are pleomorphic, they have a locally ordered lattice of glycoprotein spikes that protrude $18 \mathrm{~nm}$ from the membrane (FIG. 1 b). The spikes are predicted to comprise trimers of $\mathrm{Gn}-\mathrm{Gc}$ heterodimers and form a unique tripodlike arrangement on the virus surface (FIG. 1c). This is distinct from the tetrameric square-like lattice of hantavirus particles ${ }^{18}$ and the icosahedral arrangement of pentamers and hexamers of phlebovirus particles ${ }^{19}$. Acidification of BUNV particles results in a structural change in the head region of the spike that presumably involves the larger Gc protein, which is the predicted fusion protein (see below).

Organization of the viral genome. Like all members of the Bunyaviridae family, the genome of orthobunyaviruses comprises three unique segments of singlestranded RNA (ssRNA) that have a common organization that includes a coding region, which is flanked by $3^{\prime}$ and $5^{\prime}$ UTRs. The average size of each genome segment is generally consistent within a genus, and those of orthobunyaviruses are about $6.9 \mathrm{~kb}$ for the $\mathrm{L}$ (large) segment, $4.5 \mathrm{~kb}$ for the $\mathrm{M}$ (medium) segment and $1.0 \mathrm{~kb}$ for the $\mathrm{S}$ (small) segment ${ }^{20}$ (FIG. 2). The terminal nucleotides at the $3^{\prime}$ and $5^{\prime}$ ends of each segment are complementary, which permits the formation of a 'panhandle' structure that functions as the promoter for both the transcription and replication of each segment ${ }^{21-23}$. Although the lengths of each coding region, and hence the sizes of encoded proteins, are relatively conserved among different viruses, there is much more diversity in the length and sequence of the UTRs. In addition to functioning as promoters, the UTRs are also required for the encapsidation of the genomic RNA by the $\mathrm{N}$ protein, the termination of mRNA transcription and packaging of the RNP into virus particles ${ }^{24-27}$, and it is remarkable that these common functions are mediated by such disparate sequences. Intriguingly, deletion of internal sequences in the UTRs of BUNV resulted in attenuation of virus replication and loss of cytopathogenicity in mammalian cell culture, but the mechanistic basis of this is unknown ${ }^{28}$.

The genomic RNA segments have a negative-sense polarity, which means that the genomic RNA must be transcribed into positive-sense mRNAs for translation, and this occurs immediately after infection. The $\mathrm{S}$ segment encodes the $\mathrm{N}$ protein, the $\mathrm{M}$ segment encodes the two external glycoproteins Gn and Gc, and the L segment encodes the RdRp ${ }^{29}$. The glycoproteins are encoded as a precursor polyprotein that also contains the non-structural protein NSm. The coding order of the M segment mRNA is $\mathrm{Gn}-\mathrm{NSm}-\mathrm{Gc}^{30}$, and it is co-translationally processed by host proteases to produce the three proteins. The S segment of most orthobunyaviruses encodes a second non-structural protein, NSs, which is within the $\mathrm{N}$ protein-coding sequence and is translated from the same mRNA using an alternative AUG initiation $\operatorname{codon}^{31}$ (in a process known as leaky ribosomal scanning). However, in some orthobunyaviruses, the NSs ORF is severely truncated or even absent $t^{32,33}$. By contrast, a recently characterized orthobunyavirus, Brazoran virus, has a larger S segment (which is $1.7 \mathrm{~kb}$ ) and encodes the NSs protein in an ORF that precedes the $\mathrm{N}$ protein $\mathrm{ORF}^{34}$. To further emphasize the increasing diversity of the bunyavirus family, the newly described mosquito-associated viruses (Herbert-like viruses) that are mentioned above do not encode either NSs or $\mathrm{NSm}^{10}$. 
Haemagglutination inhibition

An immunological test to

measure the ability of

antibodies to block the

agglutination of red blood

cells by a virus. For

orthobunyaviruses, the viral

glycoproteins mediate

haemagglutination, and

antibodies to these proteins

that inhibit haemagglutination

are used to define serogroups.

Cryoelectron microscopy

A technique that preserves

the native state of biological material in a frozen hydrated

state, which is usually achieved

by rapid freezing in liquid

ethane. Specimens are

examined in a transmission

electron microscope at ultralow

temperatures and can be

visualized without the need

for heavy-metal staining.

Subtomographic averaging

An electron microscopy

technique to visualize

structures in three dimensions

(3D). The specimen stage is

tilted at regular increments

and a series of transmission

electron images is taken, which

are then processed to generate

a high-resolution (up to $5 \mathrm{~nm}$ )

3D image.

Leaky ribosomal scanning A mechanism by which internal AUG codons in an mRNA are used to initiate translation. Ribosomes occasionally skip the first AUG codon, which is often not in an optimal sequence context for initiation, and a downstream AUG is selected instead.

Cap snatching

A mechanism to prime viral mRNA synthesis. A vira endonuclease cleaves the 5 ' ends of cellular mRNAs about 12- 18 nucleotides after the 5' 7-methylguanylate residue (the cap), and the resulting short oligonucleotides are used to initiate viral mRNA transcription.

Class II fusion domain Proteins that contain this domain mediate the fusion of viral membranes to cellular membranes. Class II fusion peptides, which are also known as betapenetrenes, are found internally in the surface glycoproteins of bunyaviruses, alphaviruses and flaviviruses and mostly comprise of $\beta$-structures.

$6.9 \mathrm{~kb}$
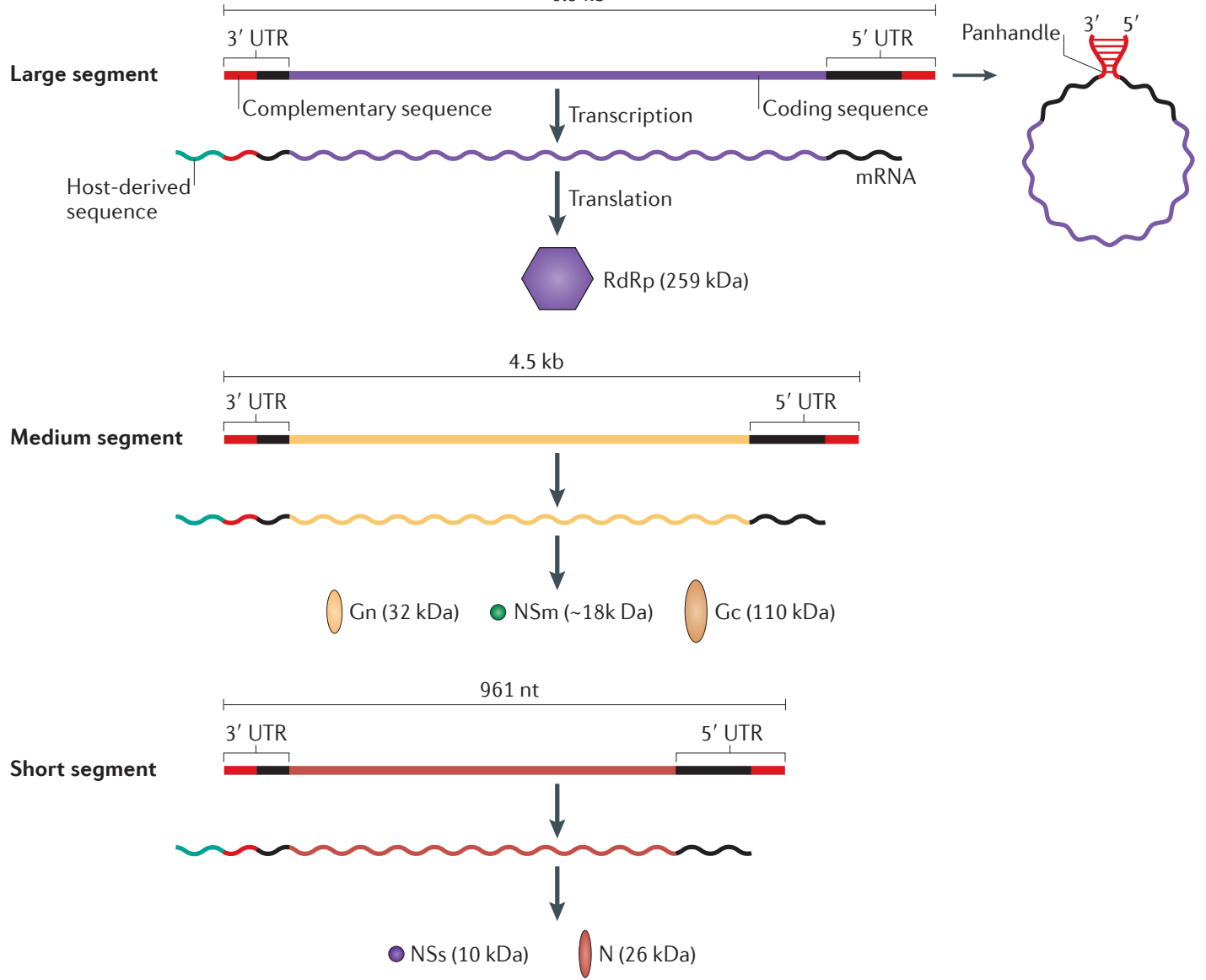

Figure 2 | Coding strategy of the orthobunyavirus genome. The small, medium and large genomic RNAs are shown and the total length of the RNA is shown above each segment, using Bunyamwera virus as an example. The genomic RNAs are transcribed into mRNAs, which contain host-derived sequences at the $5^{\prime}$ end but are truncated at the $3^{\prime}$ end compared with the template. The coding regions are shown as different coloured boxes. The terminal sequences of each genome segment are complementary (red), which results in base pairing between the $3^{\prime}$ and $5^{\prime}$ ends to form a panhandle that is used as a promoter for transcription and replication. Protein products with their estimated molecular weights are also shown. The L (large) segment encodes the L protein, which is the RNA-dependent RNA polymerase (RdRp). The M (medium) segment encodes a polyprotein that is proteolytically cleaved to generate the viral glycoproteins Gn and Gc and the non-structural protein, NSm. The S (small) segment encodes the N (nucleocapsid) protein and a second non-structural protein (NSs), which is translated from an overlapping reading frame in the same mRNA by leaky ribosomal scanning.

$L$ protein. The $\mathrm{L}$ protein that is encoded by the large genomic segment is the RdRp, and it is responsible for catalysing both transcription and replication. Conserved amino acid sequence motifs have been identified in the $\mathrm{L}$ protein, and their crucial role in RNA synthesis has been confirmed by mutagenesis ${ }^{35}$. The aminoterminal domain of the $\mathrm{L}$ protein contains an endonuclease domain that cleaves capped oligonucleotides from the $5^{\prime}$ ends of host mRNAs, which are then used to prime viral mRNA synthesis ${ }^{36}$. This process, which is known as cap snatching, is also a feature of influenza virus transcription, but in contrast to influenza viruses, for orthobunyaviruses it occurs in the cytoplasm rather than in the nucleus ${ }^{37,38}$.

Gn and Gc proteins. Orthobunyavirus glycoproteins typically differ in size: $\mathrm{Gn}$ is $32-35 \mathrm{kDa}$, whereas $\mathrm{Gc}$ is $110-120 \mathrm{kDa}$. Both proteins are type I integral membrane proteins that are modified by $N$-linked glycosylation and are rich in cysteine residues (which constitute about $5 \%$ of the total number of amino acids). Conservation of cysteine localization in the glycoproteins of different viruses suggests that they are involved in the formation of intermolecular and intramolecular disulphide bonds, which is important for protein structure and function. Gn and Gc form a heterodimer in the ER, and their transport to the Golgi complex depends on a targeting signal that has been mapped to the transmembrane domain of $\mathrm{Gn}$ in BUNV ${ }^{39}$. When Gc is expressed alone, it is retained in the ER, which suggests that $\mathrm{Gn}$ functions as a chaperone for the correct trafficking of Gc to the Golgi complex before viral budding ${ }^{39}$. Gc is predicted to contain a class II fusion domain ${ }^{40}$, and mutation of this motif in LACV supports this prediction ${ }^{41}$. Mutation of residues around the fusion peptide in BUNV Gc led to a severe reduction in membrane fusion, whereas deletion 


\section{Box 2 | Reverse genetics of orthobunyaviruses}

The ability to introduce specific changes into a viral genome by 'reverse genetics' has revolutionized virology as it has enabled the study of viral gene function in unprecedented detail. Recombinant DNA techniques are used to introduce mutations into cDNA copies of RNA virus genomes, but it is then a challenge to convert the DNA back into a viable RNA genome. A further complication for negative-sense RNA viruses is the necessity to recreate the ribonucleoprotein complex as the functional template for replication. These problems have mostly been overcome by using bacteriophage RNA polymerases (such as the T7 polymerase) to transcribe RNA from cDNA clones, using ribozymes that enable exact trimming of RNA transcripts and expressing the viral $\mathrm{N}$ (nucleocapsid) protein concomitantly with cDNA transcription to enable co-transcriptional encapsidation to form ribonucleoprotein complexes. The most widely used reverse genetic system for orthobunyaviruses was developed for Bunyamwera virus (BUNV) ${ }^{131,132}$ and was recently adapted for use with Schmallenberg virus (SBV) ${ }^{13,63}$ (see the figure). A cell line that constitutively expresses bacteriophage T7 RNA polymerase is transfected with a mixture of three transcription plasmids that contain full-length cDNA copies of each genome segment, which are cloned between the T7 promoter and the hepatitis delta ribozyme sequence. After transfection, positive-sense RNA is transcribed and processed to generate antigenome-like transcripts. These transcripts, although neither 5'-capped nor 3'-polyadenylated, can function as mRNAs for the translation of all viral proteins that, in turn, associate with the cDNA-derived RNAs to initiate the infectious cycle. 'Helper' plasmids, which contain just the viral protein-coding sequences under control of the T7 promoter (and often an internal ribosome entry sequence to ensure efficient translation) can also be transfected to express the $N$ protein and the $L$ protein (which is the RNA-dependent RNA polymerase (RdRp)) (see the figure). For example, recovery of BUNV was successful whether or not helper plamids were transfected ${ }^{132}$, whereas recovery of viable La Crosse virus was only achieved following transfection of just the three transcription plasmids ${ }^{62}$.

Variations to the system include: transcription plasmids based on cellular RNA polymerase I promoter sequences and helper plasmids that use RNA polymerase II promoters ${ }^{133}$; and provision of T7 polymerase via a co-transfected plasmid ${ }^{13}$. It is key to success that the sequence of the cDNA clone is an exact match for a functional viral genome sequence and that the ratios of viral RNAs to viral proteins in the transfected cell are appropriate to support viral replication and transcription.

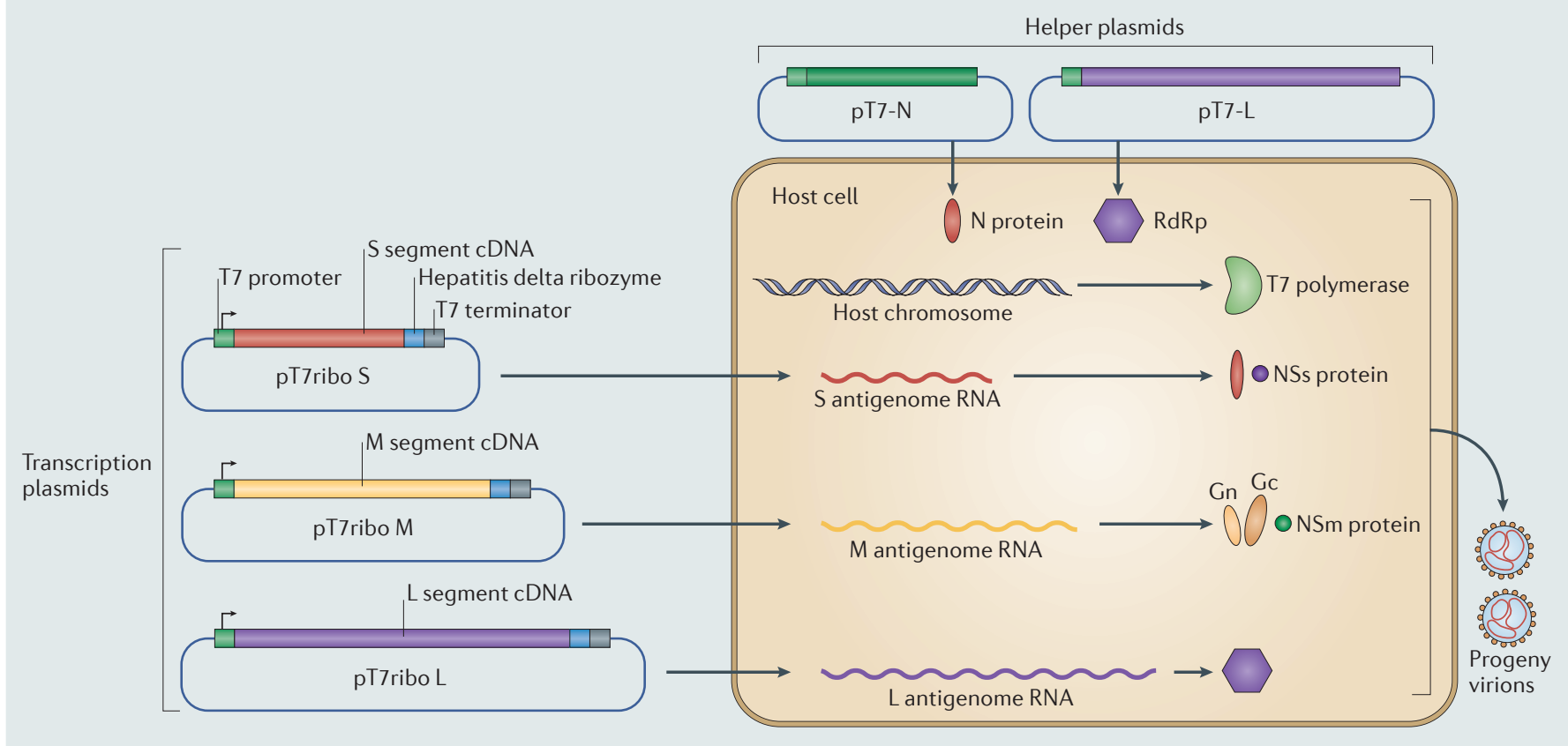

of the N-terminal domain of the Gc ectodomain had only minimal effects on virus replication ${ }^{42}$. Indeed, it has been possible to generate a recombinant virus that expresses a Gc protein with an N-terminal-truncation that is fused to GFP using reverse genetics ${ }^{43}$ (BOX 2).

N protein. The N protein, which is around $25-30 \mathrm{kDa}$ in size, is the main protein that is produced in infected cells; it is highly immunogenic and elicits complement-fixing antibodies. The $\mathrm{N}$ protein encapsidates both genomic and antigenomic RNA (but not viral mRNA) to form RNPs that are the templates for the viral RdRp. The signal for encapsidation is located in the $5^{\prime}$ end of both the genome and antigenome, and it is likely that the N protein binds to ssRNA rather than double-stranded RNA (dsRNA; such as that formed in the panhandle structure)
(FIG. 2) in a structure-dependent manner ${ }^{24,25,44}$. In addition to interacting with RNA, the $\mathrm{N}$ protein interacts with itself and also with RdRp, Gn and $\mathrm{Gc}^{45}$, although the molecular details of these other interactions are currently lacking.

The atomic structures of four orthobunyavirus N proteins (from BUNV, LACV, Leanyer virus and SBV) were recently solved, either as apoproteins or in complex with RNA ${ }^{46-51}$ (FIG. 3). These structures show that the N protein has a unique fold that is not seen in other negative-sense viruses or in other Bunyaviridae genera, such as phleboviruses (for example, Rift Valley fever virus) or nairoviruses (for example, Crimean Congo haemorrhagic fever virus $)^{52}$. The $\mathrm{N}$ protein protomer consists of two major domains (an N-terminal domain and a carboxy-terminal domain) that have flexible N-terminal 


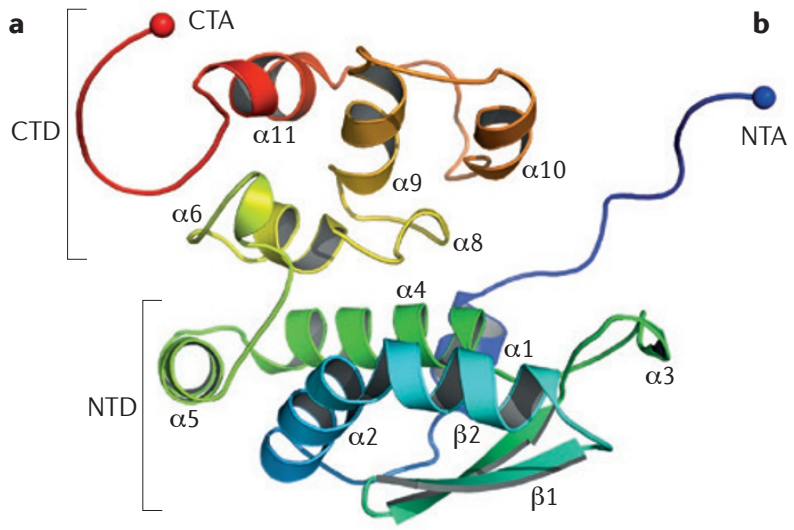

b NTA
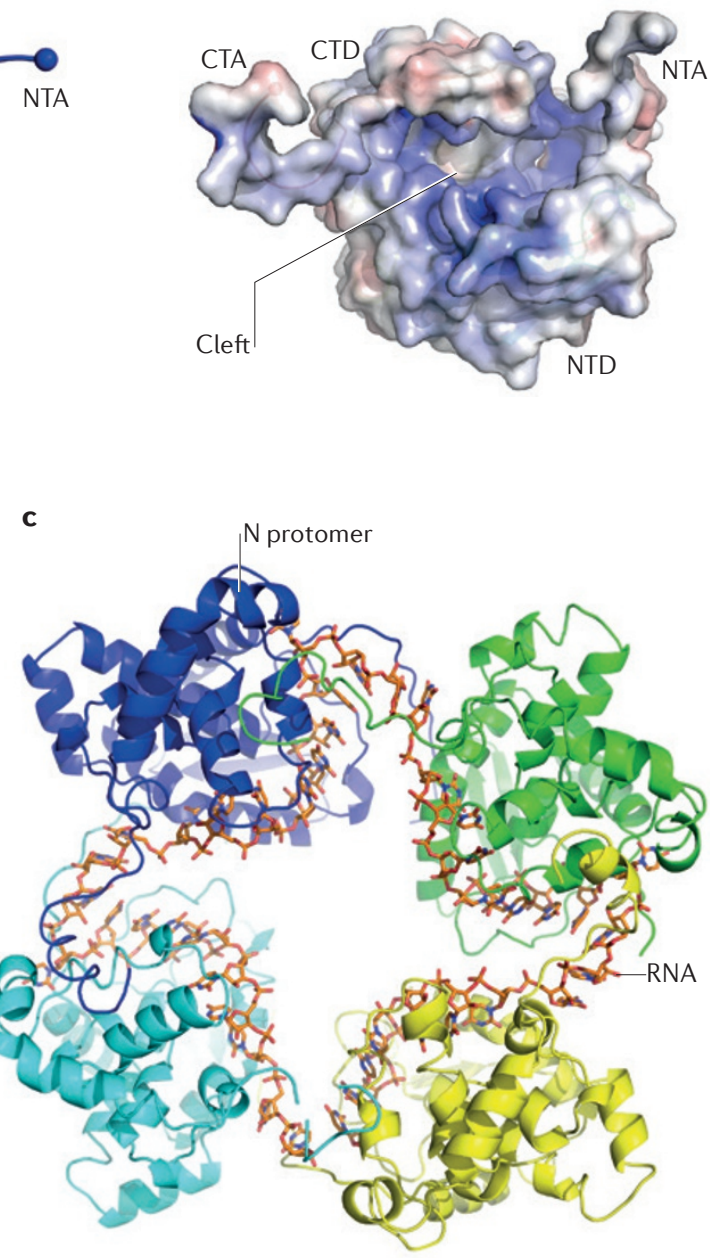

Figure 3 | Structure of the orthobunyavirus $\mathbf{N}$ protein. a| The crystal structure of the $\mathrm{N}$ (nucleocapsid) protein protomer from Schmallenberg virus (SBV) shows that it consists of an amino-terminal arm (NTA), an N-terminal domain (NTD), a carboxy-terminal domain (CTD) and a C-terminal arm (CTA). The NTA and CTA are important for mediating interactions between $\mathrm{N}$ protein monomers. b | Electrostatic surface potential map of the N protomer from SBV. Positively charged residues are shown in blue and negatively charged residues are shown in red. The central cleft that binds to viral RNA is indicated. $\mathrm{c}$ |Structure of the SBV N protein-RNA complex. The N protein forms a tetramer that binds the RNA in a cross-like shape on the inside of the tetramer. The $\mathrm{N}$ protein tetramer is shown in ribbon form, with each protomer in a different colour. The RNA is shown in stick form in orange. Parts $\mathbf{a}$ and $\mathbf{b}$ of the figure from Journal of Virology, 2013, 87, 5593-5601, http://dx.doi.org/10.1128/JVI.00223-13, reproduced with permission from American Society for Microbiology. Part c is modified, with permission, from REF. 46, Cold Spring Harbor Laboratory Press.

and C-terminal arms and a central positively charged cleft that binds the viral RNA (FIG. 3a,b). The protein forms a tetramer, in which each monomer interacts with neighbouring molecules in a head-to-tail manner that is mediated by the $\mathrm{N}$ - and C-terminal arms, and the central cleft of each monomer is aligned with that of its neighbour to create a continuous RNA-binding groove in the interior of the $\mathrm{N}$ protein ring, which binds the RNA in a cross-shape configuration (FIG. 3c). Each protomer binds to 11 bases: 8 bases in the cleft and 3 bases near the $\mathrm{N}$-terminal arm. The bound RNA is thus protected, and a local rearrangement of the $\mathrm{N}$ protomers would be required for the RdRp to access it for transcription and replication. By comparing the apoN and $\mathrm{N}$ protein-RNA complex structures from SBV, conformational changes in both the $\mathrm{N}$ - and C-terminal arms and in some of the RNA-binding residues in the RNAbinding groove were observed, which suggests how such reorganization might occur ${ }^{46,48}$ - the challenge now is to show this experimentally. Electron microscopy has shown that the orthobunyavirus RNP has a highly ordered helical appearance ${ }^{48,50,51}$, which correlates with the structure of a particular crystallographic form of the LACV N protein ${ }^{51}$. In addition to these structural studies, mutational studies of the $\mathrm{N}$ proteins from BUNV and
SBV have provided functional data that are consistent with the structural information ${ }^{47,53,54}$. Furthermore, the strict requirement of the $\mathrm{N}$ - and C-terminal arms for the oligomerization of the $\mathrm{N}$ protein provides a target for the development of small molecules that inhibit these interactions.

Non-structural proteins. Orthobunyaviruses encode one or, more commonly, two non-structural proteins. $\mathrm{NSm}$ is a $16-18 \mathrm{kDa}$ protein that is encoded between $\mathrm{Gn}$ and Gc in the glycoprotein precursor (FIG. 2). The BUNV NSm protein was shown to localize to the Golgi complex independently of other viral proteins $\mathrm{s}^{55,56}$ and to interact with the $\mathrm{C}$ terminus of $\mathrm{Gn}$. The localization of NSm to the site of virus maturation suggests that NSm may be involved in virus assembly and budding ${ }^{56}$. Consistent with this, high-resolution microscopy of infected cells has revealed that virus factories contain novel tubular structures that comprise cellular proteins and the NSm protein $^{57}$. However, the intact NSm protein is not essential for virus viability, as recombinant viruses that contain deletions within NSm or that express GFP-tagged NSm are viable, although they grow slower than the wild-type virus and to lower titres in mammalian cells ${ }^{45}$. In addition, insertion of $\beta$-galactosidase sequences into 
Minigenome

A genome analogue that comprises the $3^{\prime}$ and 5' UTRs of a viral genome segment and contains a reporter gene (for example, luciferase) in the negative-sense in place of the viral coding sequence.

\section{DC-SIGN}

(Dendritic cell-specific

intercellular adhesion molecule 3-grabbing non-integrin).

A calcium-dependent carbohydrate-binding protein (C-type

lectin) receptor that is present on the surface of both

macrophages and dendritic cells.

\section{Macropinocytosis}

A process that involves the uptake of molecules into cells in a clathrin-independent manner. Membrane ruffles trap fluid as they fold over themselves at the cell surface and undergo fusion, which results in vesicles that pinch off the membrane surface and hence deliver cargo to the cytoplasm.

\section{Caveolar endocytosis}

A clathrin-independent mechanism of endocytosis Entry into cells is mediated by caveolae, which are flask-shaped invaginations on the cell surface that contain the protein caveolin.

Rabbit reticulocyte lysate An in vitro or cell-free translation system that is based on nuclease-treated lysates of reticulocytes (the cells that are responsible for the production of

haemoglobin), which enables the translation of exogenous RNAs.
NSm of LACV did not affect the processing of the glycoprotein precursor or intracellular targeting of the mature glycoproteins to the Golgi complex ${ }^{58}$. The $\mathrm{N}$ terminus of NSm functions as an internal signal sequence for NSm itself, but it can also substitute for the signal sequence of $\mathrm{Gc}$ in viruses that have extensive deletions at the NSm-Gc gene boundary ${ }^{43}$.

Most orthobunyaviruses encode another nonstructural protein called NSs, which is around $10 \mathrm{kDa}$ in size. In contrast to the $\mathrm{N}$ protein, the amino acid sequence of NSs tends to be more variable between different viruses, and the protein is mostly hydrophobic and localizes to both the cytoplasm and the nucleus ${ }^{59}$. The NSs protein has multiple functions in the viral replication cycle and is the major virulence factor: recombinant viruses in which NSs expression is silenced are severely attenuated in animal models ${ }^{60}$. However, NSs is not essential for growth, as mutant viruses that lack NSs can replicate in mice and in cell culture ${ }^{13,61-63}$. In mammalian cell culture, NSs inhibits translation, and deletion mutants have an impaired ability to block protein synthesis; however, in mosquito cells, NSs has no effect on protein production ${ }^{61,63,64}$. NSs proteins are also involved in apoptosis, but the effects of the BUNV and LACV proteins seem to differ. For BUNV, NSs delays apoptosis ${ }^{65}$, whereas the NSs protein of LACV and other California serogroup viruses seems to induce apopto$\operatorname{sis}^{62,66}$. These NSs proteins have amino acid similarity to a region in Drosophila spp. Reaper protein, which is a known inducer of apoptosis ${ }^{67}$.

In minigenome assays, NSs inhibits RNA synthesis ${ }^{68,69}$, although the importance of this during natural infection has not yet been explored; however, it could be a mechanism to reduce the production of viral RNA transcripts and thereby block immune detection (see below).

\section{Replication cycle}

Cell entry involves interactions between the glycoproteins $\mathrm{Gn}$ and/or Gc and cell surface receptors (FIG. 4); the receptor (or receptors) are unknown, although it has been reported that Germiston orthobunyavirus entry is promoted by DC-SIGN ${ }^{70}$. Although DC-SIGN is highly expressed on dermal dendritic cells and is a good candidate for entry following transmission by an arthropod bite, other receptors are probably involved, as these viruses infect a wide range of tissues. Studies with OROV and LACV have shown that entry is likely to occur by clathrin-mediated endocytosis ${ }^{71,72}$, and for LACV, neither macropinocytosis nor caveolar endocytosis is required for entry. Furthermore, LACV entry is dependent on Rab5 but not on Rab7 (Rab5 and Rab7 are GTPases that are key regulators for the transport of endocytic vesicles to early and late endosomes, respectively), which indicates that trafficking to early endosomes is required for uncoating (FIG. 4). Several studies have suggested that Gc is the primary protein involved in attachment to both vertebrate and invertebrate cells ${ }^{73}$, but it has also been suggested that the smaller protease-resistant Gn protein of LACV functions as the attachment protein for mosquito cells $\mathrm{s}^{74,75}$ - these conflicting results need to be revisited.
Transcription. After release of the RNPs into the cytoplasm, the first event is primary transcription (FIG. 4), which involves primer-dependent synthesis of viral mRNAs and results in mRNAs that contain 12-18 hostderived nucleotides at their $5^{\prime}$ end ${ }^{37}$. Although this sequence is generally heterogeneous, a preference for a certain motif at the junction of the primer and the viral mRNA has been observed, whereby the nucleotides at the $3^{\prime}$ end of the scavenged primer are similar to those at the $5^{\prime}$ terminus of the viral RNA. It has been suggested that, after the initial transcription of two or three nucleotides, the nascent mRNA could realign backwards on the template as a consequence of the terminal sequence repeats, before further elongation resumes, which results in partial reiteration of the $5^{\prime}$ sequence ${ }^{76}$.

Orthobunyavirus mRNAs do not have detectable poly(A) tracts at their 3' ends, and no U-rich tract (which is used to signal polyadenylation by other negative-sense viruses) is present in the genome. The $3^{\prime}$ ends of the viral mRNAs have the potential to form stem-loop structures that may be involved in promoting efficient translation in a poly(A)-binding protein-independent manner ${ }^{77}$. Detailed mapping of transcription termination of the S segment of BUNV showed that the pentanucleotide sequence $5^{\prime}$-UGUCG-3' signalled termination ${ }^{26,27}$. Although this sequence is present in the BUNV L segment and in the S segments of some other orthobunyaviruses, it does not occur in the BUNV M segment, which indicates that transcription termination signals are not universally conserved.

Bunyavirus transcription requires on-going translation in the host cell, which is a unique feature among negative-sense RNA viruses ${ }^{78}$. When infected cells are treated with protein synthesis inhibitors, such as cycloheximide, essentially no full-length viral mRNAs are produced ${ }^{79}$, and in vitro transcriptase reactions yield only short RNA products, unless they are supplemented with rabbit reticulocyte lysate $e^{80,81}$. Further experiments have shown that ongoing protein synthesis is needed to prevent premature termination of transcription, and it was postulated that in the absence of functional ribosomes the nascent mRNA and the template RNA hybridize, which prevents progression of the $\mathrm{RdRp}^{82}$. This model has been tested using BUNV genome analogues that contain premature stop codons, which showed that translation of the nascent mRNA prevented premature termination ${ }^{83}$. This coupling of translation and transcription is also a feature of bacteria and archaea but is rare in eukaryotes; thus, it is unclear why this family of viruses has adopted such a mechanism.

Genome replication. The template for genome replication is full-length positive-sense RNA (known as the antigenome), which is produced in a primerindependent manner from the negative-sense genome (FIG. 4). The molecular basis for the switch from transcription to replication is unclear, but the level of $\mathrm{N}$ protein in the cell is important, as sufficient $\mathrm{N}$ protein must be available to encapsidate nascent antigenomes and genomes during replication. Only the viral RdRp and $\mathrm{N}$ proteins are required for transcription and replication ${ }^{84}$, 


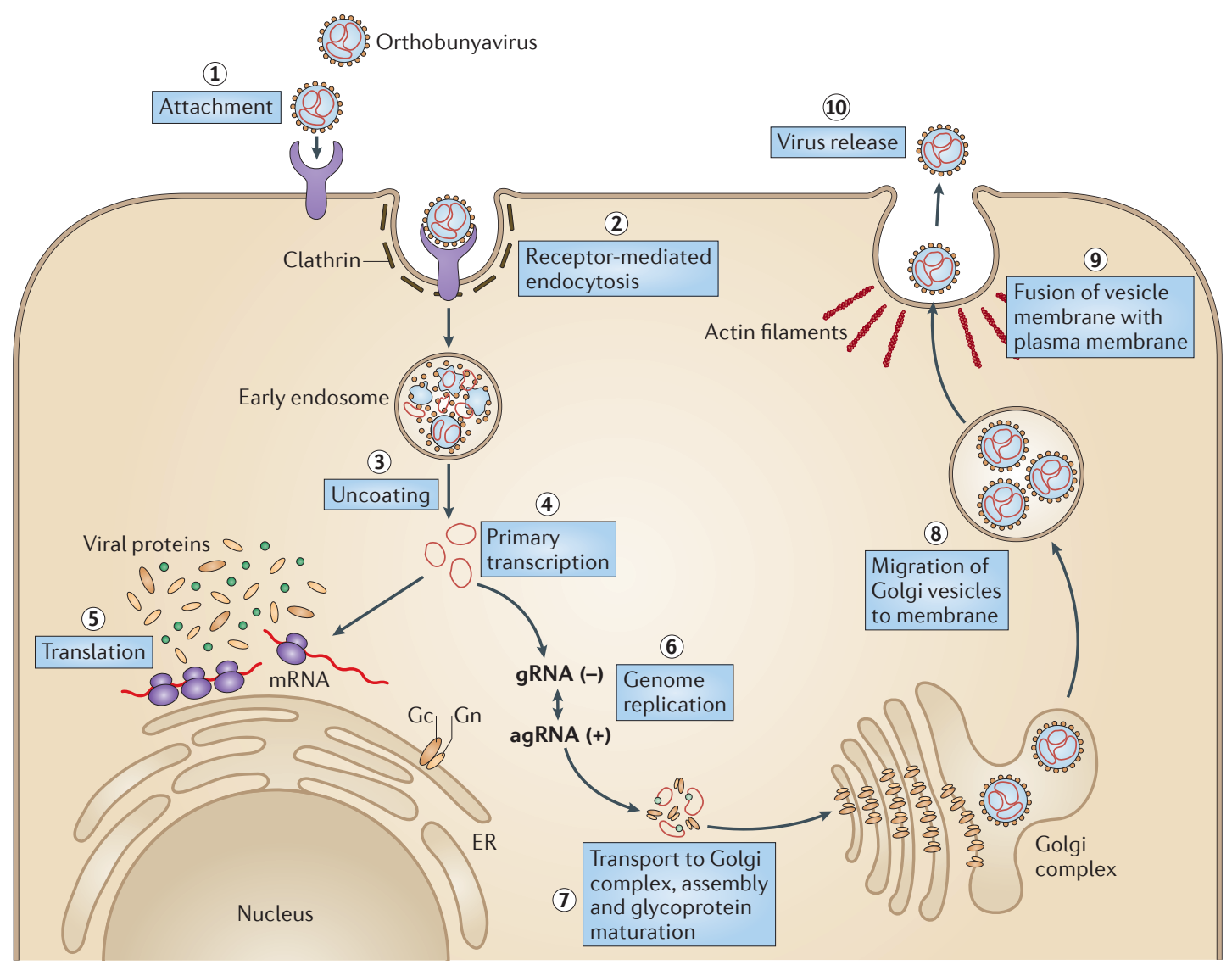

Figure 4 | The orthobunyavirus replication cycle. Replication begins with viral attachment to host receptors (step 1), which is mediated by either or both of the viral glycoproteins (Gc and Gn), followed by virus internalization via receptor-mediated endocytosis (step 2). Acidification of endocytic vesicles leads to virion uncoating (step 3) and fusion of the viral membrane with the endosomal membrane. The viral RNA-dependent RNA polymerase (RdRp) catalyses primary transcription of viral mRNAs (step 4), which are primed by host cell-derived primers (not shown). Following translation of the viral mRNAs (step 5), the glycoproteins Gn and Gc dimerize in the ER and localize to the Golgi complex using a signal in the transmembrane domain in $\mathrm{Gn}$. The three negative-sense viral genome segments (genomic RNA (gRNA)) are converted into positive-sense antigenomes (antigenomic RNA (agRNA)) for genome replication in the virus factory (step 6). The ribonucleoproteins (RNPs) are then transported to membranes of the Golgi complex that have been modified by insertion of $\mathrm{Gn}$ and $\mathrm{Gc}$, and virus particles bud into Golgi membrane-derived vesicles (step 7). Golgi vesicles that contain virus particles are trafficked to the cell surface (step 8 ) and fusion of the vesicular membranes with the plasma membrane leads to the release of infectious virions, which may be facilitated by actin filaments (step 9). Nascent viral particles are subsequently released from the cell (step 10).

Virus factory

A region of the host cell that is modified by the virus, such that it recruits cellular organelles and functions as a scaffold for virus assembly.

\section{Endoglycosidase $\mathrm{H}$}

(Endo H). An enzyme that cleaves asparagine-linked high-mannose glycans from glycoproteins but does not digest complex oligosaccharide side chains. EndoH sensitivity is used as a marker to monitor oligosaccharide processing of a protein as it transits through the Golgi complex. and whether different forms of the polymerase - for example, those that have been modified by association with different cellular factors - carry out these different activities has not been determined. The antigenome is always found encapsidated by the $\mathrm{N}$ protein in the cell, which might prevent interactions with the template for transcription, as well as minimizing the formation of dsRNA in the cell, which is important to avoid detection by the host immune response (see below). In cells that are co-infected with two different orthobunyaviruses, progeny virions may contain genome segments that are derived from both parents; this process is known as genome reassortment and can lead to reassortant viruses that have altered biological properties (BOX 3).

Assembly and budding at the Golgi complex. The viral glycoproteins $\mathrm{Gn}$ and $\mathrm{Gc}$ are targeted to, and accumulate in, the Golgi complex, where assembly occurs. Viral
RNPs are transported to regions of the Golgi membrane that have been modified by the insertion of the glycoproteins, which promotes budding into Golgi-derived vesicles (FIG. 4). Using a virus-like particle production assay, direct interactions between the cytoplasmic tails of the glycoproteins and the RNP were found to be necessary for budding ${ }^{85}$. Inside the Golgi stacks, annular particles are observed, which are defined as immature precursors that transform into dense intermediate particles $^{86,87}$. This maturation is accompanied by a change in the sensitivity of $\mathrm{Gn}$ and $\mathrm{Gc}$ to endoglycosidase $\mathrm{H}$ (endo $\mathrm{H}$ ), from complete sensitivity to partial resistance ${ }^{87,88}$, which indicates that the terminal sugar residues on the glycoproteins are processed during transport through the Golgi stacks to the trans-Golgi.

Similarly to other viruses that have segmented genomes, orthobunyaviruses have the problem of packaging a single copy of each of the three genomic segments 


\section{Box 3 | Orthobunyavirus evolution and reassortment of genome segments}

Like all negative-sense RNA viruses, the orthobunyavirus RNA-dependent RNA polymerase (RdRp) lacks a proof-reading function and therefore generates considerable genetic heterogeneity in virus populations. Furthermore, possession of a segmented genome facilitates more dramatic genetic changes owing to the ability to reassort genome segments during co-infections, which is well documented in the emergence of pandemic strains of influenza virus. During co-infection with two different bunyaviruses, reassortment can generate six potential reassortant viruses in addition to progeny that are identical to the parent viruses (see the figure). However, there are limitations to reassortment, such that only closely related viruses (for example, those within a serogroup) can reassort with each other, and there are further restrictions regarding the incompatibility of certain genome segment combinations. In a study of Bunyamwera (BUNV) serogroup viruses, the L (large) segment and S (small) segment seem to be genetically linked ${ }^{134}$ (that is, they were always inherited as a pair), although no such linkage was observed in California serogroup viruses ${ }^{135}$. The molecular basis for restriction has yet to be elucidated, but this presumably involves interactions between the $N$ (nucleocapsid) protein, the RdRp and the viral RNA.

Mosquitoes that are infected with two viruses probably provide the best opportunity for genetic mixing to occur, as mosquitoes can feed on different vertebrates that could be infected with distinct viruses. However, there seems to be a relatively short window for a mosquito to become infected by a second virus, and after 2-3 days, the mosquito is resistant to infection during a subsequent blood meal ${ }^{136}$.

Reassortants can have substantially altered phenotypic traits; for example, the $M$ (medium) segment gene products ( $\mathrm{Gn}, \mathrm{Gc}$ and $\mathrm{NSm}$ ) have a major influence on vector competence, which is broadly defined as the ability of a particular arthropod species to support efficient transmission of the virus ${ }^{137}$. A reassortant virus with a different $M$ segment could therefore be transmitted by a different arthropod vector, and in turn, the new arthropod might have different biological behaviour, leading to the possible introduction of the reassortant virus into a new host. An extreme example of phenotypic change is provided by Ngari virus, which was shown to be a reassortant virus that comprises the $L$ segment and $S$ segment of $B U N V$ and the $M$ segment of Batai virus (BATV) ${ }^{138}$. BUNV and BATV both infect humans but cause only self-limiting febrile illness; by contrast, Ngari virus is associated with severe haemorrhagic disease.

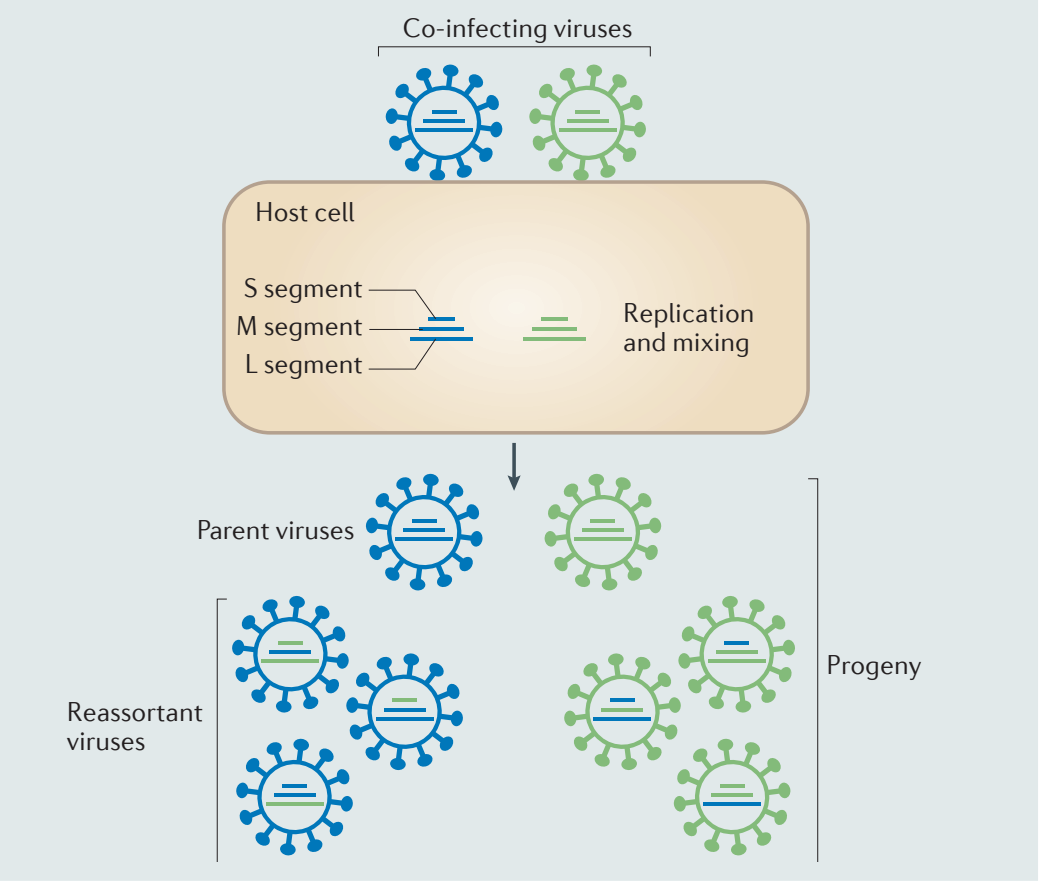

Exocytic pathway

The exocytic pathway consists of a series of membrane-bound organelles that transport proteins and lipids in vesicles from the ER to the cell surface. in order to generate an infectious particle. Genome packaging has been extensively studied in influenza virus $^{89}$, but comparatively little is known about this process in orthobunyaviruses. However, packaging seems to require only the UTR sequences, and the inclusion of
$\mathrm{N}$ protein-coding sequences did not improve packaging efficiency ${ }^{24}$. In addition, the generation of a viable recombinant BUNV that contains a hybrid $\mathrm{M}-\mathrm{L}$ segment (in which $\mathrm{M}$ segment UTRs flank the L segment ORF) would argue against the presence of packaging signals that extend into the coding sequence ${ }^{90}$. These findings differ from those in influenza virus, in which both non-coding and coding sequences are used as packaging signals.

Virus release. The progeny virions are transported to the cell surface via the exocytic pathway, which has been visualized using a recombinant BUNV that expresses GFP-tagged $\mathrm{Gc}^{43}$. Following virus release, a second maturation step occurs, during which the viral spikes (that is, the $\mathrm{Gn}$ and Gc trimers) become more defined ${ }^{86,87}$. Correlative light and electron microscopy has shown that multilamellar structures and extracellular membranedeficient filament bundles are generated in infected cells at times of maximal virus release ${ }^{91}$. Progeny virions were found to be associated with the filament bundles (which contain actin), and cytochalasin D treatment of infected cells, which disrupts actin filaments, reduced released virus titres by $\sim 100$-fold. These data suggest that actin filaments are involved in BUNV egress and could also be involved in transport of the virus to uninfected neighbouring cells.

\section{Persistent infection of arthropod cells}

In contrast to the usually lytic infection of vertebrate cells, orthobunyavirus infection of arthropod cells results in the establishment of a persistent infection. The cellular interactions between the virus and the host that enable efficient viral replication without harming the vector are unknown. Some clues come from studies of persistent infection of cultured mosquito cells, which have been described for several orthobunyaviruses ${ }^{29,92}$. The production of $\mathrm{L}$ segmentderived defective-interfering particles ${ }^{93}$ and the selflimiting production of $\mathrm{N}$ protein by encapsidation of $\mathrm{S}$ segment-derived $\mathrm{mRNA}^{94}$ have been suggested to contribute to persistence. Studies of BUNV mutants that lack NSs have shown that there is little impairment in their ability to replicate in certain Aedes albopictus cells (such as C6/36 and C7-10, which lack a functional Dicer 2-mediated RNAi response) but that NSs is important for infection of the A. albopictus U4.4 cell line (which has an active Dicer 2 enzyme) and also for efficient infection and spread out of the midgut of Aedes aegypti mosquitoes ${ }^{95}$. This suggests that a balance between the innate defence that is mounted by the RNAi pathway and virus replication must be achieved in the invertebrate host, but whether NSs directly disrupts the RNAi system requires further investigation. Although infected mosquito cells are not killed by the infection, transient morphological changes are observed $^{95,96}$, and extensive filopodial-like structures occur in cells during the early phases of infection. The presence of virus particles in these projections suggests a mechanism for cell-to-cell spread of virus without rupture of the cell membrane. 


\section{Evasion of innate immunity in vertebrate cells}

Pattern recognition receptors (PRRs) recognize foreign molecules, known as pathogen-associated molecular patterns (PAMPs), and in the case of viruses, these include various forms of nucleic acid, such as dsRNA and 5'-triphosphorylated RNA, which are recognized intracellularly by two cytoplasmic RNA helicases, retinoic acid-inducible gene I (RIGI) and melanoma differentiation-associated protein 5 (MDA5) ${ }^{97}$. Binding of an RNA ligand to these PRRs activates a signalling cascade that induces the expression of several transcription factors (including IFN regulatory factor 3 (IRF3), nuclear factor- $\kappa \mathrm{B}(\mathrm{NF}-\kappa \mathrm{B})$ and cyclic AMP-dependent transcription factor (ATF2)-c-Jun), which promote transcription of interferon- $\beta$ (IFN $\beta$ ), leading to the upregulation of hundreds of IFN-stimulated genes (ISGs), some of which have antiviral activity ${ }^{98}$. It was recently shown that RIGI recognizes the nucleocapsid of LACV, which contains the terminal panhandle with a $5^{\prime}$ triphosphate, and recognition occurs in the absence of transcription or replication ${ }^{99}$. This suggests that the innate immune response is triggered at the earliest stage of infection, just after viral entry. Little is known about the antiviral activities of most ISG products, although they probably inhibit viral replication at many stages of the virus life cycle. The protein MxA inhibits LACV and BUNV replication by sequestering newly synthesized $\mathrm{N}$ protein in cytoplasmic inclusions, which blocks replication ${ }^{100}$. Other ISGs that inhibit BUNV include PKR, RSAD2 (commonly known as viperin), MTAP44, IFITM3, IRF1, HPSE and UBE2L6 (REFS 101,102,103). Individually, the degree of inhibition by these ISGs is relatively modest, but they presumably function together such that their combined effects are highly restrictive.

The IFN response is extremely powerful at controlling virus replication and, in cells that have been pretreated with IFN (to induce the antiviral state), orthobunyavirus replication is restricted, but the addition of IFN after the infection is established has little effect on virus replication ${ }^{102}$. In mammalian cells, BUNV, LACV and SBV NSs proteins inhibit the induction of IFN ${ }^{61,63,104,105}$, and for BUNV and LACV, this occurs downstream of transcription factor activation. BUNV NSs prevents phosphorylation of serine 2 in the heptad repeat of the C-terminal domain (CTD) of RNA polymerase II, which leads to an arrest in mRNA elongation and 3 ' processing, thereby causing a general inhibition of cellular transcription ${ }^{59}$. This inhibition seems to be mediated by interactions between the C terminus of NSs and the Mediator component MED8 (REF. 106). MED8 is in the head module of Mediator, which interacts directly with the CTD of RNA polymerase II (REF. 107). The N terminus of BUNV NSs is also involved in transcriptional inhibition, although the mechanism involved remains to be elucidated ${ }^{108}$. Similarly, NSs of LACV targets RNA polymerase II, but in this case, the protein triggers proteasomal degradation of the hyperphosphorylated polymerase (that is, the transcriptionally active form) by exploiting early events in the cellular DNA damage response pathway $^{109}$.

\section{Outlook}

Important progress has been made in recent years on several aspects of orthobunyavirus biology, particularly advances in determining the structures of individual viruses and virus proteins, which are providing new insights into the infection and replication processes, and the interactions between viruses and both vertebrate and arthropod hosts. However, there is much more to learn about this fascinating group of viruses. We are only just beginning to appreciate the evolutionary relationships between orthobunyaviruses: complete genome sequencing on a larger scale would be a major advance for this area, as less than one-quarter of the $\sim 170$ recognized orthobunyavirus genomes have been fully sequenced. Analysis of the available genomes indicates that reassortment (BOX 3) is more common in nature than perhaps previously appreciated, which has led to the suggestion that "most if not all bunyaviruses currently recognized may represent reassortants, some of which may be reassortants of existing viruses, and some of which may be reassortants of extinct viruses" (REF. 110). The propensity for genome reassortment, the emergence of SBV in Europe, the spread of OROV in South America and the potential for climate change to aid the emergence of other novel variants highlight that increased global surveillance, together with improved diagnostic methods, are required to monitor orthobunyavirus activity; for example, the establishment of sentinel herds and active vector trapping at international ports and airports would aid early detection efforts ${ }^{111}$.

Besides vaccines against Simbu serogroup viruses ${ }^{112}$, including the recent development of two inactivated vaccines that have been approved for $\mathrm{SBV}^{113}$, no vaccines or antiviral agents are available against orthobunyaviruses. However, vaccine manufacturers have little incentive to develop vaccines against human orthobunyaviruses owing to a perceived limited market. Similarly, there has been little interest in research on antiviral drugs. The availability of efficient reverse genetics systems (BOX 2) could be exploited for vaccine development, and several studies have shown that attenuated viruses can be engineered by the introduction of deletions within non-coding sequences, the rearrangement of coding and non-coding sequences, the deletion of the NSs protein and mutations in the $\mathrm{N}$ protein ${ }^{28,54,61,90,105}$. The construction of such attenuated strains could form the basis for live attenuated vaccines, and by combining such alterations, it might be possible to generate a virus that is unable to revert to virulence via back-mutation. In addition, it should be possible to generate viruses that are unable to reassort (BOX 3) with circulating wild-type strains, which would limit their propensity to evolve. Second, the determination of orthobunyavirus $\mathrm{N}$ protein structures provides opportunities for the rational design of small-molecule inhibitors to interfere with $\mathrm{N}$ protein function. Understanding how individual ISGs inhibit orthobunyavirus replication could also be exploited to develop effective inhibitory compounds ${ }^{98}$.

Another area of orthobunyavirus research that is likely to expand in the coming years is focused on developing a 
detailed understanding of the interaction with the arthropod vector, such as the molecular basis for vector competence, and how this knowledge might also be exploited for infection control. This will require further advances in genomics and reagent development for vector species.
Arthropods have their own innate immune defences, which are mostly based on RNAi for protection against viruses ${ }^{114}$, and how the viruses can survive these defences and replicate without harming the vector requires further investigation.
1. Rosenberg, R., Johansson, M. A., Powers, A. M. $\delta$ Miller, B. R. Search strategy has influenced the discovery rate of human viruses. Proc. Natl Acad. Sci. USA 110, 13961-13964 (2013).

2. Vaheri, A. et al. Uncovering the mysteries of hantavirus infections. Nature Rev. Microbiol. 11, 539-550 (2013).

3. Calisher, C. H. in The Bunyaviridae (ed. Elliott, R. M.) 1-17 (Plenum Press, 1996).

4. Haddow, A. D. \& Odoi, A. The incidence risk clustering, and clinical presentation of La Crosse virus infections in the eastern United States, 2003-2007. PLOS ONE 4, e6145 (2009).

5. Beaty, B. \& Calisher, C. Bunyaviridae - natural history. Curr. Top. Microbiol. Immunol. 169, 27-78 (1991).

6. Patrican, L. A. \& DeFoliart, G. R. Lack of adverse effect of transovarially acquired La Crosse virus infection on the reproductive capacity of Aedes triseriatus (Diptera: Culicidae). J. Med. Entomol. 22, 604-611 (1985).

7. Jackson, B. T., Brewster, C. C. \& Paulson, S. L. La Crosse virus infection alters blood feeding behavior in Aedes triseriatus and Aedes albopictus (Diptera: Culicidae). J. Med. Entomol. 49, 1424-1429 (2012).

8. Reese, S. M., Beaty, M. K., Gabitzsch, E. S., Blair, C. D. $\&$ Beaty, B. J. Aedes triseriatus females transovarially infected with La Crosse virus mate more efficiently than uninfected mosquitoes. J. Med. Entomol. 46, 1152-1158 (2009).

9. Thompson, W. H. \& Beaty, B. J. Venereal transmission of La Crosse (California encephalitis) arbovirus in Aedes triseriatus mosquitoes. Science 196, 530-531 (1977).

10. Marklewitz, M. et al. Discovery of a unique novel clade of mosquito-associated bunyaviruses. J. Virol. 87 12850-12865 (2013)

11. Taylor, K. G. \& Peterson, K. E. Innate immune response to La Crosse virus infection. J. Neurovirol. 20, 150-156 (2014)

12. Bennett, R. S. et al. La Crosse virus infectivity, pathogenesis, and immunogenicity in mice and monkeys. Virol. J. 5, 25 (2008).

13. Varela, M. et al. Schmallenberg virus pathogenesis, tropism and interaction with the innate immune system of the host. PLoS Pathog. 9, e1003133 (2013).

14. Wernike, K. et al. Schmallenberg virus experimental infection of sheep. Vet. Microbiol. 166, 461-466 (2013).

15. Barrett, A. D. T. \& Shope, R. E. in Topley and Wilson's Microbiology and Microbial Infections. (eds Mahy, B. W. J. \& Meulen, V.t.) 1025-1058 (Hodder Arnold, 2005).

16. Obijeski, J. F., Bishop, D. H., Murphy, F. A. \& Palmer, E. L. Structural proteins of La Crosse virus. J. Virol. 19, 985-997 (1976).

17. Bowden, T. A. et al. Orthobunyavirus ultrastructure and the curious tripodal glycoprotein spike. PLOS Pathog. 9, e1003374 (2013).

This cryoelectron microscopy study of Bunyamwera virus particles presents the three-dimensional structure of the glycoprotein spike resolved to $3 \mathrm{~nm}$.

18. Battisti, A. J. et al. Structural studies of Hantaan virus J. Virol. 85, 835-841 (2011).

19. Sherman, M. B., Freiberg, A. N., Holbrook, M. R. \& Watowich, S. J. Single-particle cryo-electron microscopy of Rift Valley fever virus. Virology 387. 11-15 (2009).

20. Plyusnin, A et al. in Virus taxonomy: Ninth Report of the International Committee on Taxonomy of Viruses. (eds King, A. M. Q., Adams, M. J., Carstens, E. B. \& Lefkowits, E. J.) 725-741 (Elsevier, 2012).

21 Barr, J. N., Elliott, R. M., Dunn, E. F. \& Wertz, G. W. Segment-specific terminal sequences of Bunyamwera bunyavirus regulate genome replication. Virology 311 326-338 (2003)

22. Barr, J. N. \& Wertz, G. W. Role of the conserved nucleotide mismatch within 3'- and 5'-terminal regions of Bunyamwera virus in signaling transcription. J. Virol. 79, 3586-3594 (2005).
23. Kohl, A., Dunn, E. F., Lowen, A. C. \& Elliott, R. M Complementarity, sequence and structural elements within the $3^{\prime}$ and $5^{\prime}$ non-coding regions of the Bunyamwera orthobunyavirus S segment determine promoter strength. J. Gen. Virol. 85, 3269-3278 (2004).

24. Kohl, A., Lowen, A. C., Leonard, V. H. J. \& Elliott, R. M. Genetic elements regulating packaging of the Bunyamwera orthobunyavirus genome. J. Gen. Virol. 87, 177-187 (2006)

25. Osborne, J. C. \& Elliott, R. M. RNA binding properties of bunyamwera virus nucleocapsid protein and selective binding to an element in the $5^{\prime}$ terminus of the negative-sense S segment. J. Virol. 74, 9946-9952 (2000)

26. Barr, J. N., Rodgers, J. W. \& Wertz, G. W. Identification of the Bunyamwera bunyavirus transcription termination signal. J. Gen. Virol. 87, 189-198 (2006)

27. Blakqori, G., Lowen, A. C. \& Elliott, R. M. The small genome segment of Bunyamwera orthobunyavirus harbours a single transcription termination signal. J. Gen. Virol. 93, 1449-1455 (2012).

28. Mazel-Sanchez, B. \& Elliott, R. M. Attenuation of Bunyamwera orthobunyavirus replication by targeted mutagenesis of genomic UTRs and creation of viable viruses with minimal genome segments. J. Virol. 86, 13672-13678 (2012)

29. Elliott, R. M. \& Blakqori, G. in Bunyaviridae. Molecular and Cellular Biology (eds Plyusnin, A. \& Elliott, R. M.) 1-39 (Caister Academic Press, 2011).

30. Fazakerley, J. K. et al. Organization of the middle RNA segment of snowshoe hare Bunyavirus. Virology 167 422-432 (1988)

31. Fuller, F., Bhown, A. S. \& Bishop, D. H. Bunyavirus nucleoprotein, N, and a non-structural protein, NSS, are coded by overlapping reading frames in the S RNA J. Gen. Virol. 64, 1705-1714 (1983).

32. Mohamed, M., McLees, A. \& Elliott, R. M. Viruses in the Anopheles A, Anopheles B, and Tete serogroups in the Orthobunyavirus genus (family Bunyaviridae) do not encode an NSs protein. J. Virol. 83, 7612-7618 (2009)

33. Chowdhary, R. et al. Genetic characterization of the Wyeomyia group of orthobunyaviruses and their phylogenetic relationships. J. Gen. Virol. 93 1023-1034 (2012)

34. Lanciotti, R. S. et al. Isolation of a novel orthobunyavirus (Brazoran virus) with a $1.7 \mathrm{~kb} \mathrm{~S}$ segment that encodes a unique nucleocapsid protein possessing two putative functional domains. Virology 444, 55-63 (2013)

35. Jin, H. \& Elliott, R. M. Mutagenesis of the L protein encoded by Bunyamwera virus and production of monoclonal antibodies. J. Gen. Virol. 73, 2235-2244 (1992).

36. Reguera, J., Weber, F. \& Cusack, S. Bunyaviridae RNA polymerases (L-protein) have an $\mathrm{N}$-terminal, influenza-like endonuclease domain, essential for viral cap-dependent transcription. PLoS Pathog. 6 e 1001101 (2010)

This paper characterizes the endonuclease domain that is located in the $\mathbf{N}$ terminus of the orthobunyavirus RdRp, which generates the capped primers that are required for viral mRNA synthesis.

37. Patterson, J. L., Holloway, B. \& Kolakofsky, D. La Crosse virions contain a primer-stimulated RNA polymerase and a methylated cap-dependent endonuclease. J. Virol. 52, 215-222 (1984).

38. Rossier, C., Patterson, J. \& Kolakofsky, D. La Crosse virus small genome mRNA is made in the cytoplasm. J. Virol. 58, 647-650 (1986).

39. Shi, X., Lappin, D. F. \& Elliott, R. M. Mapping the Golgi targeting and retention signal of Bunyamwera virus glycoproteins. J. Virol. 78, 10793-10802 (2004).

This study shows that the Golgi-targeting signal is present in the transmembrane domain of the orthobunyavirus $\mathrm{Gn}$ protein.
40. Garry, C. E. \& Garry, R. F. Proteomics computational analyses suggest that the carboxyl terminal glycoproteins of Bunyaviruses are class II viral fusion protein ( $\beta$-penetrenes). Theor. Biol. Med. Model. 1, 10 (2004).

41. Plassmeyer, M. L. et al. Mutagenesis of the La Crosse virus glycoprotein supports a role for Gc (1066-1087) as the fusion peptide. Virology 358, 273-282 (2007).

42. Shi, X., Goli, J., Clark, G., Brauburger, K. \& Elliott, R. M. Functional analysis of the Bunyamwera orthobunyavirus Gc glycoprotein. J. Gen. Virol. 90, 2483-2492 (2009).

43. Shi, X., van Mierlo, J. T., French, A. \& Elliott, R. M Visualizing the replication cycle of bunyamwera orthobunyavirus expressing fluorescent protein-tagged Gc glycoprotein. J. Virol. 84, 8460-8469 (2010). This paper describes the generation of a recombinant BUNV in which the Gc glycoprotein is fused to GFP, which enables viral propagation to be monitored by light microscopy.

44. Ogg, M. M. \& Patterson, J. L. RNA binding domain of Jamestown Canyon virus S segment RNAs. J. Virol. 81 13754-13760 (2007).

45. Shi, X. et al. Requirement of the $\mathrm{N}$-terminal region of orthobunyavirus nonstructural protein NSm for virus assembly and morphogenesis. J. Virol. 80 8089-8099 (2006).

46. Dong, H., Li, P., Bottcher, B., Elliott, R. M. \& Dong, C. Crystal structure of Schmallenberg orthobunyavirus nucleoprotein-RNA complex reveals a novel RNA sequestration mechanism. RNA 19, 1129-1136 (2013).

47. Dong, H., Li, P., Elliott, R. M. \& Dong, C. Structure of Schmallenberg orthobunyavirus nucleoprotein suggests a novel mechanism of genome encapsidation. J. Virol. 87, 5593-5601 (2013).

48. Ariza, A. et al. Nucleocapsid protein structures from orthobunyaviruses reveal insight into ribonucleoprotein architecture and RNA polymerization. Nucleic Acids Res. 41, 5912-5926 (2013).

49. Li, B et al. Bunyamwera virus possesses a distinct nucleocapsid protein to facilitate genome encapsidation. Proc. Natl Acad. Sci. USA 110 9048-9053 (2013)

50. Niu, F. et al. Structure of the Leanyer orthobunyavirus nucleoprotein-RNA complex reveals unique architecture for RNA encapsidation. Proc. Natl Acad. Sci. USA 110, 9054-9059 (2013).

51. Reguera, J., Malet, H., Weber, F. \& Cusack, S. Structural basis for encapsidation of genomic RNA by La Crosse orthobunyavirus nucleoprotein. Proc. Nat Acad. Sci. USA 110, 7246-7251 (2013). References 47, 48, 49, 50 and 51 report crystal structures of orthobunyavirus nucleocapsid proteins.

52. Zheng, W. \& Tao, Y. J. Genome encapsidation by orthobunyavirus nucleoproteins. Proc. Natl Acad. Sci. USA 110, 8769-8770 (2013).

53. Leonard, V. H., Kohl, A., Osborne, J. C., McLees, A. \& Elliott, R. M. Homotypic interaction of Bunyamwera virus nucleocapsid protein. J. Virol. 79, 13166-13172 (2005).

54. Eifan, S. A. \& Elliott, R. M. Mutational analysis of the Bunyamwera orthobunyavirus nucleocapsid protein gene. J. Virol. 83, 11307-11317 (2009).

55. Nakitare, G. W. \& Elliott, R. M. Expression of the Bunyamwera virus $M$ genome segment and intracellular localisation of NSm. Virology 195 511-520 (1993)

56. Lappin, D. F., Nakitare, G. W., Palfreyman, J. W. \& Elliott, R. M. Localisation of Bunyamwera bunyavirus G1 glycoprotein to the Golgi requires association with G2 but not NSm. J. Gen. Virol. 75, 3441-3451 (1994).

57. Fontana, J., Lopez-Montero, N., Elliott, R. M., Fernandez, J. J. \& Risco, C. The unique architecture of Bunyamwera virus factories around the Golgi complex. Cell. Microbiol. 10, 2012-2028 (2008). 
58. Bupp, K., Stillmock, K. \& Gonzalez-Scarano, F. Analysis of the intracellular transport properties of recombinant La Crosse virus glycoproteins. Virology 220, 485-490 (1996).

59. Thomas, D. et al. Inhibition of RNA polymerase II phosphorylation by a viral interferon antagonist. J. Biol. Chem. 279, 31471-31477 (2004).

60. Eifan, S., Schnettler, E., Dietrich, I., Kohl, A. \& Blomstrom, A. L. Non-structural proteins of arthropodborne bunyaviruses: roles and functions. Viruses 5 , 2447-2468 (2013).

61. Bridgen, A., Weber, F., Fazakerley, J. K. \& Elliott, R. M. Bunyamwera bunyavirus nonstructural protein NSs is a nonessential gene product that contributes to viral pathogenesis. Proc. Natl Acad. Sci. USA 98, 664-669 (2001).

62. Blakqori, G. \& Weber, F. Efficient cDNA-based rescue of La Crosse bunyaviruses expressing or lacking the nonstructural protein NSs. J. Virol. 79, 10420-10428 (2005).

63. Elliott, R. M. et al. Establishment of a reverse genetics system for Schmallenberg virus, a newly emerged orthobunyavirus in Europe. J. Gen. Virol. 94, 851-859 (2013).

64. Hart, T. J., Kohl, A. \& Elliott, R. M. Role of the NSs protein in the zoonotic capacity of Orthobunyaviruses. Zoonoses Publ. Health 56, 285-296 (2009).

65. Kohl, A. et al. Bunyamwera virus nonstructural protein NSs counteracts interferon regulatory factor 3-mediated induction of early cell death. J. Virol. 77. 7999-8008 (2003).

66. Mukherjee, P., Woods, T. A., Moore, R. A. \& Peterson, K. E. Activation of the innate signaling molecule MAVS by bunyavirus infection upregulates the adaptor protein SARM1, leading to neuronal death. Immunity 38, 705-716 (2013).

67. Colon-Ramos, D. A. et al. Inhibition of translation and induction of apoptosis by Bunyaviral nonstructural proteins bearing sequence similarity to reaper. $\mathrm{Mol}$. Biol. Cell 14, 4162-4172 (2003).

68. Weber, F., Dunn, E. F., Bridgen, A. \& Elliott, R. M. The Bunyamwera virus nonstructural protein NSs inhibits viral RNA synthesis in a minireplicon system. Virology 281, 67-74 (2001)

69. Blakqori, G., Kochs, G., Haller, O. \& Weber, F. Functional $\mathrm{L}$ polymerase of La Crosse virus allows in vivo reconstitution of recombinant nucleocapsids. J. Gen. Virol. 84, 1207-1214 (2003).

70. Lozach, P. Y. et al. DC-SIGN as a receptor for phleboviruses. Cell Host Microbe 10, 75-88 (2011).

71. Santos, R. I. et al. Oropouche virus entry into HeLa cells involves clathrin and requires endosomal acidification. Virus Res. 138, 139-143 (2008).

72. Hollidge, B. S. et al. Orthobunyavirus entry into neurons and other mammalian cells occurs via clathrinmediated endocytosis and requires trafficking into early endosomes. J. Virol. 86, 7988-8001 (2012). This paper describes the entry mechanism of LACV into cells of the central nervous system.

73. Hacker, J. K. \& Hardy, J. L. Adsorptive endocytosis of California encephalitis virus into mosquito and mammalian cells: a role for $\mathrm{G} 1$. Virology $235,40-47$ (1997).

74. Ludwig, G. V., Christensen, B. M., Yuill, T. M. \& Schultz, K. T. Enzyme processing of La Crosse virus glycoprotein $\mathrm{G} 1$ : a bunyavirus-vector infection model. Virology 171, 108-113 (1989).

75. Ludwig, G. V., Israel, B. A., Christensen, B. M., Yuill, T. M. \& Schultz, K. T. Role of La Crosse virus glycoproteins in attachment of virus to host cells. Virology 181, 564-571 (1991).

76. Jin, H. \& Elliott, R. M. Characterisation of Bunyamwera virus S RNA that is transcribed and replicated by the $\mathrm{L}$ protein expressed from recombinant vaccinia virus. J. Virol. 67, 1396-1404 (1993).

77. Blakqori, G., van Knippenberg, I. \& Elliott, R. M Bunyamwera orthobunyavirus S-segment untranslated regions mediate poly(A) tail-independent translation. J. Virol. 83, 3637-3646 (2009)

78. Kolakofsky, D., Bellocq, C. \& Raju, R. The translational requirement for La Crosse virus S-mRNA synthesis. Cold Spring Harb. Symp. Quant. Biol. 52, 373-379 (1987).

79. Patterson, J. L. \& Kolakofsky, D. Characterization of La Crosse virus small-genome transcripts. J. Virol. 49, 680-685 (1984).

80. Vialat, P. \& Bouloy, M. Germiston virus transcriptase requires active $40 \mathrm{~S}$ ribosomal subunits and utilizes capped cellular RNAs. J. Virol. 66, 685-693 (1992).

1. Bellocq, C., Raju, R., Patterson, J. \& Kolakofsky, D. Translational requirement of La Crosse virus S-mRNA ynthesis: in vitro studies. J. Virol 61, 87-95 (1987)

82. Raju, R. \& Kolakofsky, D. Translational requirement of La Crosse virus S-mRNA synthesis: in vivo studies. J. Virol. 61, 96-103 (1987).

83. Barr, J. N. Bunyavirus mRNA synthesis is coupled to translation to prevent premature transcription termination. RNA 13, 731-736 (2007). This study explores the unique requirement for ongoing protein synthesis for orthobunyavirus mRNA synthesis and demonstrates that the translation of nascent mRNAs prevents premature termination of viral transcription.

84. Dunn, E. F., Pritlove, D. C., Jin, H. \& Elliott, R. M Transcription of a recombinant bunyavirus RNA template by transiently expressed bunyavirus proteins. Virology 211, 133-143 (1995).

85. Shi, X., Kohl, A., Li, P. \& Elliott, R. M. Role of the cytoplasmic tail domains of Bunyamwera orthobunyavirus glycoproteins $\mathrm{Gn}$ and $\mathrm{Gc}$ in virus assembly and morphogenesis. J. Virol. 81, 10151-10160 (2007)

86. Salanueva, I. J. et al. Polymorphism and structural maturation of Bunyamwera virus in Golgi and postGolgi compartments. J. Virol. 77, 1368-1381 (2003)

87. Novoa, R. R., Calderita, G., Cabezas, P., Elliott, R. M. $\bar{\varnothing}$ Risco, C. Key Golgi factors for structural and functional maturation of bunyamwera virus. J. Virol. 79, 10852-10863 (2005)

88. Madoff, D. H. \& Lenard, J. A membrane glycoprotein that accumulates intracellularly: cellular processing of the large glycoprotein of LaCrosse virus. Cell $\mathbf{2 8}$ 821-829 (1982)

89. Hutchinson, E. C., von Kirchbach, J. C., Gog, J. R. \& Digard, P. Genome packaging in influenza A virus. J. Gen. Virol. 91, 313-328 (2010)

90. Lowen, A. C., Boyd, A., Fazakerley, J. K. \& Elliott, R. M. Attenuation of bunyavirus replication by rearrangement of viral coding and noncoding sequences. J. Virol. 79, 6940-6946 (2005).

91. Sanz-Sanchez, L. \& Risco, C. Multilamellar structures and filament bundles are found on the cell surface during bunyavirus egress. PLOS ONE 8, e65526 (2013).

92. Borucki, M. K., Kempf, B. J., Blitvich, B. J., Blair, C. D. \& Beaty, B. J. La Crosse virus: replication in vertebrate and invertebrate hosts. Microbes Infect. 4, 341-350 (2002).

93. Scallan, M. F. \& Elliott, R. M. Defective RNAs in mosquito cells persistently infected with Bunyamwera virus. J. Gen. Virol. 73, 53-60 (1992)

94. Hacker, D., Raju, R. \& Kolakofsky, D. La Crosse virus nucleocapsid protein controls its own synthesis in mosquito cells by encapsidating its mRNA. J. Virol. 63 , 5166-5174 (1989)

This study shows that the dsRNA panhandle structure in LACV nucleocapsids triggers the innate immune response by activating RIGI shortly after viral entry.

95. Szemiel, A. M., Failloux, A. B. \& Elliott, R. M. Role of Bunyamwera orthobunyavirus NSs protein in infection of mosquito cells. PLoS Negl Trop. Dis. 6, e1823 (2012)

96. Lopez-Montero, N. \& Risco, C. Self-protection and survival of arbovirus-infected mosquito cells. Cell. Microbiol. 13, 300-315 (2011).

97. Randall, R. E. \& Goodbourn, S. Interferons and viruses: an interplay between induction, signalling antiviral responses and virus countermeasures. J. Gen. Virol. 89, 1-47 (2008)

98. Schoggins, J. W. \& Rice, C. M. Interferon-stimulated genes and their antiviral effector functions. Curr. Opin. Virol. 1, 519-525 (2011).

99. Weber, M. et al. Incoming RNA virus nucleocapsids containing a 5'-triphosphorylated genome activate RIG-I and antiviral signaling. Cell Host Microbe 13 336-346 (2013)

100. Kochs, G. Janzen, C., Hohenberg, H. \& Haller, O. Antivirally active MxA protein sequesters La Crosse virus nucleocapsid protein into perinuclear complexes. Proc. Natl Acad. Sci. USA 99, 3153-3158 (2002).

101. Streitenfeld, H. et al. Activation of PKR by Bunyamwera virus is independent of the viral interferon antagonist NSs. J. Virol. 77, 5507-5511 (2003)

102. Carlton-Smith, C. \& Elliott, R. M. Viperin, MTAP44, and protein kinase $\mathrm{R}$ contribute to the interferoninduced inhibition of Bunyamwera orthobunyavirus replication. J. Virol. 86, 11548-11557 (2012).
103. Schoggins, J. W. et al. Pan-viral specificity of IFNinduced genes reveals new roles for cGAS in innate immunity. Nature 505, 691-695 (2014).

104. Weber, F. et al. Bunyamwera bunyavirus nonstructura protein NSs counteracts the induction of alpha/beta interferon. J. Virol. 76, 7949-7955 (2002).

105. Blakqori, G. et al. La Crosse bunyavirus nonstructural protein NSs serves to suppress the type I interferon system of mammalian hosts. J. Virol. 81, 4991-4999 (2007).

106. Leonard, V. H., Kohl, A., Hart, T. J. \& Elliott, R. M Interaction of Bunyamwera Orthobunyavirus NSs protein with mediator protein MED8: a mechanism for inhibiting the interferon response. J. Virol. 80, 9667-9675 (2006).

107. Poss, Z. C., Ebmeier, C. C. \& Taatjes, D. J. The Mediator complex and transcription regulation. Crit. Rev. Biochem. Mol. Biol. 48, 575-608 (2013)

108. van Knippenberg, I., Carlton-Smith, C. \& Elliott, R. M. The N-terminus of Bunyamwera orthobunyavirus NSs protein is essential for interferon antagonism. J. Gen. Virol. 91, 2002-2006 (2010).

109. Verbruggen, P. et al. Interferon antagonist NSs of La Crosse virus triggers a DNA damage response-like degradation of transcribing RNA polymerase II. J. Biol. Chem. 286, 3681-3692 (2011).

This paper describes the mechanism by which the LACV NSs protein degrades host cell RNA polymerase II to evade the innate immune response of the host.

110. Briese, T., Calisher, C. H. \& Higgs, S. Viruses of the family Bunyaviridae: are all available isolates reassortants? Virology 446, 207-216 (2013). This provocative paper discusses the importance of reassortment in bunyavirus evolution.

111. Wernike, K. et al. Schmallenberg virus - two years of experiences. Prev. Vet. Med. http://dx.doi. org/10.1016/j.prevetmed.2014.03.021 (2014)

112. Kim, Y. H. et al. Development of inactivated trivalent vaccine for the teratogenic Aino, Akabane and Chuzan viruses. Biologicals 39, 152-157 (2011).

113. Wernike, K., Nikolin, V. M., Hechinger, S., Hoffmann, B. $\&$ Beer, M. Inactivated Schmallenberg virus prototype vaccines. Vaccine 31, 3558-3563 (2013).

114. Donald, C. L., Kohl, A. \& Schnettler, E. New insights into control of arbovirus replication and spread by insect RNA interference pathways. Insects 3, 511-531 (2012).

115. Hoffmann, B. et al. Novel orthobunyavirus in cattle, Europe, 2011. Emerg. Infect. Dis. 18, 469-472

(2012)

This paper describes the initial characterization of Schmallenberg virus as a new orthobunyavirus and provides the first report of a Simbu serogroup virus in Europe.

116. van den Brom, R. et al. Epizootic of ovine congenital malformations associated with Schmallenberg virus infection. Tijdschr Diergeneeskd 137, 106-111 (2012)

117. Afonso, A et al. The Schmallenberg virus epidemic in Europe - 2011-2013. Prev. Vet. Med. http:// dx.doi.org/10.1016/j.prevetmed.2014.02.012 (2014).

118. European Commission. Statement on the Schmallenberg Virus Situation issued by the European Commission together with the EU Member States following the working group held on 17 February 2012. [online], http://ec.europa.eu/food/animal/ diseases/schmallenberg_virus/docs/statement schmallenberg 17022012 en.pdf (2012).

119. Harris, K. A. et al. The impact of Schmallenberg virus on British sheep farms during the 2011/2012 lambing season. Vet. Rec. http://dx.doi.org/10.1136/vr.102295 (2014)

120 Goller, K. V., Hoper, D., Schirrmeier, H Mettenleiter, T. C. \& Beer, M. Schmallenberg virus as possible ancestor of Shamonda virus. Emerg. Infect. Dis. 18, 1644-1646 (2012)

121. Veronesi, E. et al. Implicating Culicoides biting midges as vectors of Schmallenberg virus using semiquantitative RT-PCR. PLOS ONE 8, e57747 (2013).

22. Sedda, L. \& Rogers, D. J. The influence of the wind in the Schmallenberg virus outbreak in Europe. Sci. Rep. 3, 3361 (2013).

This study models the contribution of wind to the spread of Schmallenberg virus and suggests that most infections originate from the bite of midges that are spread by downwind movements.

23. Bilk, S. et al. Organ distribution of Schmallenberg virus RNA in malformed newborns. Vet. Microbiol. 159, 236-238 (2012). 
124. Kupferschmidt, K. Scientists rush to find clues on new animal virus. Science 335, 1028-1029 (2012).

125. Larska, M., Lechowski, L., Grochowska, M. ¿ Zmudzinski, J. F. Detection of the Schmallenberg virus in nulliparous Culicoides obsoletus/scoticus complex and $C$. punctatus - the possibility of transovarial virus transmission in the midge population and of a new vector. Vet. Microbiol. 166, 467-473 (2013).

126. Meroc, E. et al. Follow-up of the Schmallenberg virus seroprevalence in Belgian cattle. Transbound. Emerg. Dis. http://dx.doi.org/10.1111/tbed.12202 (2013).

127. Hulst, M. et al. Genetic characterization of an atypical Schmallenberg virus isolated from the brain of a malformed lamb. Virus Genes 47, 505-514 (2013)

128. Rosseel, T. et al. DNase SISPA-next generation sequencing confirms Schmallenberg virus in Belgian field samples and identifies genetic variation in Europe. PLOS ONE 7, e41967 (2012).

129. Coupeau, D. Claine, F., Wiggers, L., Kirschvink, N. \& Muylkens, B. In vivo and in vitro identification of a hypervariable region in Schmallenberg virus. J. Gen. Virol. 94, 1168-1174 (2013).
130. Fischer, M. et al. A mutation 'hot spot' in the Schmallenberg virus M segment. J. Gen. Virol. 94 1161-1167 (2013)

131. Bridgen, A. \& Elliott, R. M. Rescue of a segmented negative-strand RNA virus entirely from cloned complementary DNAs. Proc. Natl Acad. Sci. USA 93 15400-15404 (1996).

This paper describes the initial reverse genetics system for Bunyamwera virus, which was the first system used to generate a negative-sense RNA virus with a segmented genome from plasmid DNAs.

132. Lowen, A. C., Noonan, C., McLees, A. \& Elliott, R. M. Efficient bunyavirus rescue from cloned cDNA. Virology 330, 493-500 (2004)

133. Ogawa, Y., Sugiura, K., Kato, K., Tohya, Y. \& Akashi, H Rescue of Akabane virus (family Bunyaviridae) entirely from cloned cDNAs by using RNA polymerase I. J. Gen. Virol. 88, 3385-3390 (2007).

134. Iroegbu, C. U. \& Pringle, C. R. Genetic interactions among viruses of the Bunyamwera complex. J. Virol. 37, 383-394 (1981).

135. Urquidi, V. \& Bishop, D. H. Non-random reassortment between the tripartite RNA genomes of La Crosse and snowshoe hare viruses. J. Gen. Virol. 73, 2255-2265 (1992).

136. Beaty, B. J., Bishop, D. H., Gay, M. \& Fuller, F Interference between bunyaviruses in Aedes triseriatus mosquitoes. Virology 127, 83-90 (1983).

137. Beaty, B. J. et al. Molecular basis of bunyavirus transmission by mosquitoes: role of the middle-sized RNA segment. Science 211, 1433-1435 (1981).

138. Briese, T., Bird, B., Kapoor, V., Nichol, S. T. \& Lipkin, W. I. Batai and Ngari viruses: M segment reassortment and association with severe febrile disease outbreaks in East Africa. J. Virol. 80, 5627-5630 (2006).

\section{Acknowledgements}

Work in the author's laboratory is supported by a Wellcome Trust Senior Investigator Award. The author apologizes to colleagues whose work could not be cited owing to space limitations.

Competing interests statement

The author declares no competing interests. 